\title{
Calculation of acoustic flow-rate measurements error with the help of Fermat's principle
}

\author{
Alexander Petrov ${ }^{1, *}$ and Semyon Shkundin ${ }^{1, * *}$ \\ ${ }^{1}$ MISIS National University of Science and Technology
}

\begin{abstract}
The establishment of dispatching and automatic control systems for mine ventilation is impossible without the availability of perfect air flow rate sensors. Existing anemometers (tachometer, heat) do not meet these requirements. The error of average in cross section velocity measurements with such sensors reaches $15-20$, sometimes $30 \%$. The reason - the speed measured at one point is interpreted as the average over the cross section. The reliability of the sensors is small, because they are exposed to the damaging effect of a dusty atmosphere. Stationary installed anemometers clutter cross section, which is not always allowed. Fermat's variational principle is used for derivation of the formula for the time of propagation of a sonic signal between two set points $A$ and $B$ in a steady three-dimensional flow of a fluid or gas. It is shown that the fluid flow changes the time of signal reception by a value proportional to the flow rate independently of the velocity profile. The time difference in the reception of the signals from point $B$ to point $A$ and vice versa is proportional with a high accuracy to the flow rate. It is shown that the relative error of the formula does not exceed the square of the largest Mach number. This makes it possible to measure the flow rate of a fluid or gas with an arbitrary steady subsonic velocity field
\end{abstract}

\section{Introduction}

In our country and abroad $[1,2]$ a number of elaborations have been fulfilled targeting creation of the integrating acoustic anemometer for the mine workings. Its principle of operation is based on the fact that the acoustics impulse propagation time depends on the moving medium speed in which these waves are propagating. Recording the changes in that time, you can calculate the speed of a moving stream. Acoustic transducers are located on opposite walls of a working (Fig. 1) and do not clutter up their section. Due to the fact that the acoustic beam crosses the entire curve of the velocity distribution in the flow, the acoustic anemometer reacts on the average over the cross section velocity of the air flow with an error not exceeding $5 \%$ (according to preliminary estimation).

There are three methods for measurement of airflow rate by acoustic integrating anemometer: time-pulse, frequency and phase, each of them can be implemented as singlechannel or two-channel scheme of measurement. In the first case there is one pair of acoustic transducers (emitter and receiver), and the radiation of oscillations has only one direction -

\footnotetext{
*e-mail: petrovipmech@gmail.com

**e-mail: shkundin@mail.ru
} 


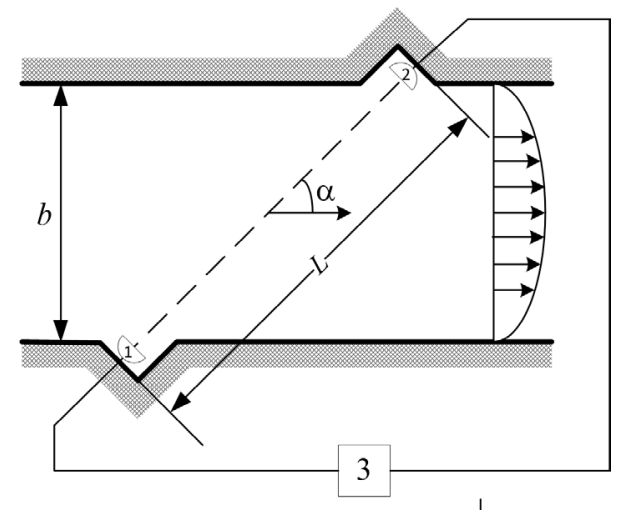

Figure 1. The arrangement of acoustics integrating anemometer transducers. 1and 2 -acoustics transducers; 3 - the electronics block

downstream or upstream; in the second case there are two pairs of transducers, i.e., radiation occurs simultaneously in two directions along the flow and against it. The two-channel scheme allows to achieve greater accuracy than single-channel. Due to the fact that the acoustic beam crosses the entire velocity distribution diagram in the flow, the acoustic anemometer measures the average velocity of the air flow with an error not exceeding 5\% (according to previous experimental estimates). In this study, we give the derivation of the theoretical estimate of such an error.
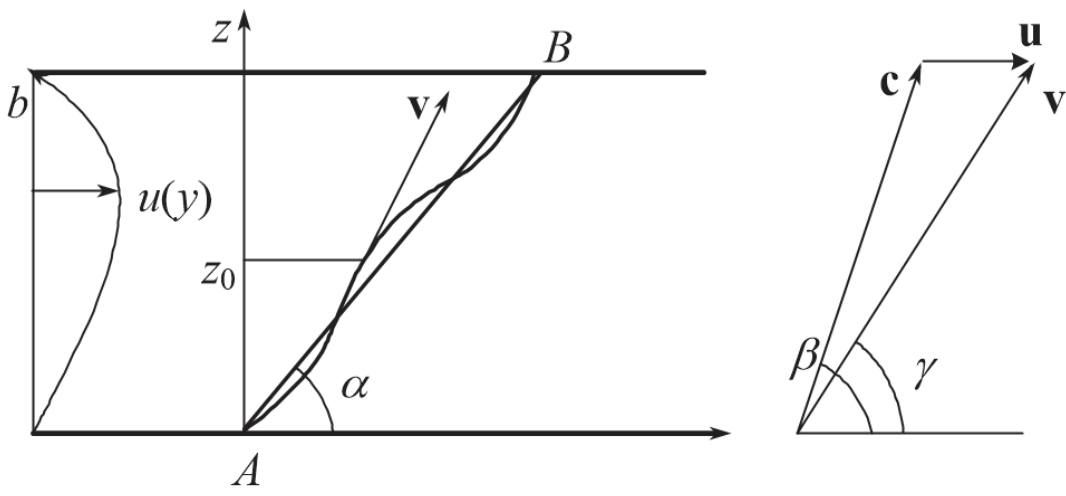

Figure 2. Schematic representation of the air flow-rate measurement in a mine (on the left). The acoustic beam vector $\mathbf{v}$ is composed of the sound velocity vector $\mathbf{c}$ and the airflow velocity $\mathbf{u}$ (on the right) 


\section{Propagation time of the acoustic beam between two points in a homogeneous gas flow}

The dependence of the acoustic signal time intervals difference of transmission from point $A$ to point $B$ and backwards on the velocity $u$ of the homogeneous air flow can be derived as follows.

We direct the $x$ axis along the velocity and the $z$ axis perpendicular to the velocity. Let the point $A$ be arranged at the origin of the Cartesian coordinate system $x, z$, and the point $B$ have the coordinates $a, b$. If the air is at rest, the sonic signal with the velocity $c$ passes the distance $A B$ in the time $t_{0}=\sqrt{a^{2}+b^{2}} / c$ propagating along the shortest straight-line path $A B$.

In the presence of a homogeneous air flow $u=$ const, the sonic signal also passes the path $A B$ along the shortest straight line, but with the velocity $v$ dependent on the air-flow velocity. The velocity is $v$ determined as follows. We found the angle $\beta-\gamma$ between the vectors $c$ and $v$ by the theorem of $\operatorname{sines} \sin (\beta-\gamma) / u=\sin \gamma / c$ and, using it, expressed the angle $\beta$ through $\gamma$ (see Fig. 2, on the right). From here, we obtain

$$
\sin (\beta-\gamma)=M \sin \gamma, \cos \beta=\cos \gamma\left(1-M^{2} \sin ^{2} \gamma\right)^{1 / 2}-M \sin ^{2} \gamma, M=u / c .
$$

The length of the vector $\mathbf{v}$ is determined by the cosine theorem $v^{2}=c^{2}+u^{2}+2 c u \cos \beta$. The signal-receive time is $t_{A B}=\sqrt{a^{2}+b^{2}} / v$, the Mach-number expansion of which has the form

$$
t_{A B}=t_{0}+\frac{1}{c}\left(-a M+\frac{2 a^{2}+b^{2}}{2 \sqrt{a^{2}+b^{2}}} M^{2}-a M^{3}\right) .
$$

This formula can be used for measuring of the air velocity $u$. For this purpose, we determined the difference $\Delta t=t_{B A}-t_{A B}$ of the signal-reception times along the inverse trajectory of the beam from point $B$ to point $A$ and along the direct trajectory from point $A$ to point $B$. The times $t_{A B}$ and $t_{B A}$ differ in the sign of the Mach number, and the terms of the expansions in the odd powers of the Mach number have opposite signs and coincide for the even powers. Therefore, the expansion of $\Delta t$ includes terms of only odd powers:

$$
\begin{gathered}
\Delta t=\frac{2 a}{c}\left(M+M^{3}+O\left(M^{4}\right)\right)=\frac{2 a}{b c^{2}} Q\left(1+M^{2}+O\left(M^{4}\right)\right) \\
Q=\int_{0}^{b} u(y) d y=b u
\end{gathered}
$$

Thus, for a homogeneous flow, the flow rate $Q$ is measured through the difference of time pulses by the formula

$$
Q=\frac{b c^{2}}{2 a} \Delta t(1+\Delta)
$$

The relative error amounts to a value equal to the square of the Mach number $\Delta=-M^{2}+$ $O\left(M^{4}\right)$. Formula (2) is the basic of the time-pulse method of air flow rate measuring the in mines $[1,2]$. It remains unclear how the inhomogeneity of the distribution of the velocity vector $\mathbf{u}(x, y, z)$ affects the error $\Delta$. For investigating this problem, we use Fermat's variational principle known in acoustics.

\section{Fermat's principle}

We consider an arbitrary steady gas flow with the velocity vector $\mathbf{u}(x, y, z)$, the components $u_{x}, u_{y}, u_{z}$ of which depend on the coordinates $x, y, z$. For determining the trajectory of the 
acoustic signal and the time of its arrival from point $A$ to point $B$, it is convenient to use Fermat's variational minimum principle, which is formulated as follows (see [3], p. 87): among all paths from point $A$ to point $B$, the acoustic signal passes along the real path in the shortest time.

It is convenient to set the gas flow by three dimensionless velocity components (Mach numbers).

$$
M_{x}=\frac{u_{x}(x, y, z)}{c}, M_{y}=\frac{u_{y}(x, y, z)}{c}, M_{z}=\frac{u_{z}(x, y, z)}{c},
$$

It is convenient to search for the trajectory of the acoustic beam in the parametric form $x(z), y(z), z, z \in(0, b)$. In the medium at rest, the trajectory is a straight-line segment $x=\frac{a}{b} z, y=0, z$, between two $\mathrm{b}$ points with the coordinates $A(0,0,0), B(a, 0, b)$. In the presence of the flow, the acoustic beam has a complex three-dimensional trajectory, the deviation of which from a straight line is equal to the Mach number in order of magnitude. If the Mach number is small, it depends only on the parameter $z$ up to the square of the Mach number on the beam trajectory:

$$
M_{x}=\frac{u_{x}((a / b) z, 0, z)}{c}, M_{y}=\frac{u_{y}((a / b) z, 0, z)}{c}, M_{z}=\frac{u_{z}((a / b) z, 0, z)}{c},
$$

Fermat's functional has the following analytical form:

$$
F=\int_{0}^{b} \frac{s_{z}}{v} d z, s_{z}=\sqrt{1+x_{z}^{2}+y_{z}^{2}}
$$

where $d s=s_{z} d z$ is the length element on the trajectory of the acoustic beam and $v$ is the modulus of the acoustic-beam velocity. The acoustic-beam velocity vector $v \mathbf{n}$ is composed of the sonic velocity vector $c \mathbf{m}$ and the flow velocity $c \mathbf{M}: c \mathbf{m}+c \mathbf{M}=v \mathbf{n}$, where $\mathbf{m}$ and $\mathbf{n}$ are the unit vectors. From this, we obtain the quadratic equation for determining of the velocity $v$

$$
c^{2}=(v \mathbf{n}-c \mathbf{M})^{2} \Rightarrow v^{2}-2 v c(\mathbf{n M})-c^{2}\left(1-M^{2}\right)=0, M^{2}=M_{x}^{2}+M_{y}^{2}+M_{z}^{2},
$$

Its solution has the form

$$
v=c(\mathbf{n M})+\sqrt{1+(\mathbf{n M})-M^{2}},(\mathbf{n M})=\left(s_{z}\right)^{-1}\left(M_{x} \frac{d x}{d z}+M_{y} \frac{d y}{d z}+M_{z}\right) .
$$

Substituting the expression found for $v$ into Fermat's functional, we obtain

$$
\begin{gathered}
t_{A B}=\int_{0}^{b} \frac{d z}{c} L\left(x_{z}, y_{z}, z\right), \\
L\left(x_{z}, y_{z}, z\right)=\frac{1+x_{z}^{2}+y_{z}^{2}}{M_{x} x_{z}+M_{y} y_{z}+M_{z}+\sqrt{A_{0}+A_{1}+A_{2}}} \\
A_{0}=1-M_{x}^{2}-M_{y}^{2}, A_{1}=2 M_{z}\left(M_{x} x_{z}+M_{y} y_{z}\right), \\
A_{2}=\left(1-M_{x}^{2}-M_{y}^{2}\right) x_{z}^{2}+\left(1-M_{x}^{2}-M_{y}^{2}\right) y_{z}^{2}+2 M_{x} M_{y} x_{z} y_{z} . \\
x_{z}=\frac{d x}{d z}, y_{z}=\frac{d y}{d z},
\end{gathered}
$$


We directed the $x$ axis along the average velocity of the flow. Then we obtain for the gas volume flow rate,

$$
Q=\int_{0}^{b} u_{x} d z=c b \overline{M_{x}}, \overline{M_{x}}=\frac{1}{b} \int_{0}^{b} M_{x} d z
$$

and zero $\overline{M_{y}}=\overline{M_{z}}=0$ for the average values of the Mach number components along axes $y$ and $z$.

We accept the condition that the derivative of flow-velocity components along the longitudinal coordinate $x$ is much smaller than the derivative with respect to the transverse coordinate $z$. Then the Euler equations for the extremal of the functional $t_{A B}$ take the form [4]

$$
\frac{d}{d z}\left(\frac{\partial L}{\partial x_{z}}\right)=0, \frac{d}{d z}\left(\frac{\partial L}{\partial y_{z}}\right)=0
$$

The solution of these equations with the conditions at the ends of the interval $z=0$ and $z=b$ determines the beam trajectory $x(z), y(z)$.

\section{March-number expansion}

We look for the solution to Eqs. (4) in the form of the expansions in terms of the Machnumber power. For this purpose, we represent Eqs. (4) in the form of the expansions

$$
K_{x}=\frac{\partial L}{\partial x_{z}}=\frac{x_{z}}{s_{z}}-M_{x}+L_{x 2}-M_{x} M^{2}, K_{y}=\frac{\partial L}{\partial y_{z}}=\frac{y_{z}}{s_{z}}-M_{y}+L_{y 2}-M_{y} M^{2}
$$

where $K_{x}$ and $K_{y}$ are the constants of integration and $L_{x 2}$ and $L_{y 2}$ are the terms quadratic in the Mach number.

We look for the solution to Eqs. (5) in the form of the expansions in terms of the Machnumber power and calculate them.

\section{Minimum signal-transmission time}

The signal-transmission time can be determined after substituting the expansion $L=L_{0}+$ $L_{1}+L_{2}+L_{3}$ of the integrand into formula (3):

$$
t_{A B}=t_{0}+t_{1}+t_{2}+t_{3}, \quad t_{n}=\frac{1}{c} \int_{0}^{b} L_{n} d z, \quad n=0,1,2,3
$$

It is remarkable that the difference in the time pulses is determined only by the odd terms $t_{1}$ and $t_{3}: t_{B A}-t_{A B}=-2 t_{1}-2 t_{3}$. The first term $-2 t_{1}$ corresponds to the flow-rate formula (2), and the second term determines the error

$$
\begin{gathered}
\Delta=-\frac{t_{3}}{t_{1}}=-\frac{{\overline{M_{x}}}^{3}+\overline{M_{x} M_{z}^{2}}}{\overline{M_{x}}}-\frac{a^{2}\left(-4 \overline{M_{x}^{2}} \overline{M_{x}}+4{\overline{M_{x}}}^{3}-\overline{M_{x} M_{z}^{2}}+\overline{M_{x}} M_{z}^{2}\right)}{2 b^{2} \overline{M_{x}}}- \\
-\frac{a^{4}\left(\overline{M_{x}^{3}}-3 \overline{M_{x}^{2}} \overline{M_{x}}+2{\overline{M_{x}}}^{3}-3 \overline{M_{x} M_{z}^{2}}+3 \overline{M_{x}} \overline{M_{z}^{2}}\right)}{2 b^{4} \overline{M_{x}}}-\frac{b\left(\overline{M_{x}} \overline{M_{x} M_{z}}+\overline{M_{z}^{3}}\right)}{a M_{x}}
\end{gathered}
$$


It is convenient to introduce the variances in the Mach-number distribution

$$
\overline{\Delta M^{2}}=\frac{1}{b} \int_{0}^{b}(M(y)-\bar{M})^{2} d y, \overline{\Delta M^{3}}=\frac{1}{b} \int_{0}^{b}(M(y)-\bar{M})^{3} d y=\bar{M}^{3}+3 \bar{M}, \overline{\Delta M^{2}}+\overline{\Delta M^{3}}
$$

By means of the expressions

$$
\overline{M^{2}}=\overline{(\bar{M}+\Delta M)^{2}}=\bar{M}^{2}+\overline{\Delta M^{2}}, \quad \overline{M^{3}}=\overline{\left.(\bar{M}+\Delta M)^{3}\right)}=\bar{M}^{3}+3 \bar{M} \overline{\Delta M^{2}}+\overline{\Delta M^{3}}
$$

the formula for the error takes the form

$$
\begin{gathered}
\Delta=-{\overline{M_{x}}}^{2}+2 \frac{a^{2} \overline{\Delta M_{x}^{2}}}{b^{2}}-\frac{a^{4} \overline{\Delta M_{x}^{3}}}{2 b^{4} \overline{M_{x}}}-\frac{b\left(\overline{M_{x}} \overline{M_{x} M_{z}}+\overline{M_{z}^{3}}\right)}{a \overline{M_{x}}}- \\
-{\overline{M_{z}}}^{2}-2 \frac{\overline{\Delta M_{x} M_{z}^{2}}}{\overline{M_{x}}}+\frac{a^{2} \overline{\Delta M_{x} M_{z}^{2}}}{2 b^{2} \overline{\Delta M_{x} M_{z}^{2}} M_{x}}+\frac{3 a^{4} \overline{\Delta M_{x} M_{z}^{2}}}{2 b^{4} M_{x}}
\end{gathered}
$$

We consider two particular cases. In the first case $a=b$, the formula for the error is

$$
\Delta=-{\overline{M_{x}}}^{2}+2 \overline{\Delta M_{x}^{2}}-\frac{\overline{\Delta M_{x}^{3}}}{2 \overline{M_{x}}}-\overline{M_{z}^{2}}-\frac{\overline{M_{z}^{3}}}{\overline{M_{x}}}+\frac{\overline{\Delta M_{x} M_{z}^{2}}}{\overline{M_{x}}}
$$

In the second case $a>>b$

$$
\Delta=-\frac{a^{4}\left(\overline{\Delta M_{x}^{3}}-3 \overline{\Delta M_{x} M_{z}^{2}}\right)}{2 b^{4} \overline{M_{x}}}
$$

As can be seen, the relative errors of flow-rate measering in mines are small values for any $3 \mathrm{D}$ flow of the order of the square of the average Mach number $M^{2}$.

\section{Conclusion}

The new method for average in air duct cross section flow rate measuring is proposed. The method uses formula (2) which was obtained for uniform flow. We showed that formula (2) is applicable to arbitrary $3 \mathrm{D}$ flow in mines. The relative error flow rate measuring in mines using this method for the arbitrary case has small values of the order of the square of the average Mach number $M^{2}$.

The work was supported by the Russian Science Foundation, project No 14-19-01633.

\section{References}

[1] S. Z. Shkundin, O. A. Kremleva, and V. A. Rumyantseva, Theory of Acoustic Anemometry (Izd-vo Akademii gornykh nauk, Moscow, 2001) [in Russian].

[2] Patent http://misis.ru/eiis-dost.

[3] L. D. Landau and E. M. Lifshits, Hydrodynamics (Nauka, M., 1986) [in Russian].

[4] V. I. Smirnov, Course of Higher Mathematics, Vol. IV, Part 1, Chapt. II Calculus of variations (Moscow, Nauka 1981) [in Russian]. 